\title{
Observations of interplanetary scintillation in China
}

\author{
Li-Jia Liu and Bo Peng \\ National Astronomical Observatories, Chinese Academy of Sciences \\ email: ljliu@nao.cas.cn
}

\begin{abstract}
The Sun affects the Earth in multiple ways. In particular, the material in interplanetary space comes from coronal expansion in the form of solar wind, which is the primary source of the interplanetary medium. Ground-based Interplanetary Scintillation (IPS) observations are an important and effective method for measuring solar wind speed and the structures of small diameter radio sources. In this paper we will discuss the IPS observations in China.
\end{abstract}

Keywords. solar wind, interplanetary scintillation, observation

\section{Introduction}

Radiation from a distant compact radio source is scattered by the density irregularities in the solar wind plasma and produces a random diffraction pattern on the ground. The motion of these irregularities converts this pattern into temporal intensity fluctuations which are observed as IPS. IPS observations with ground-based telescopes can estimate the solar wind velocity and also the structures of distant compact radio sources(Hewish \& Symonds 1969; Armstron \& Coles 1972). This kind of measurement, can give information on the solar wind out of the ecliptic plane and close to the Sun.

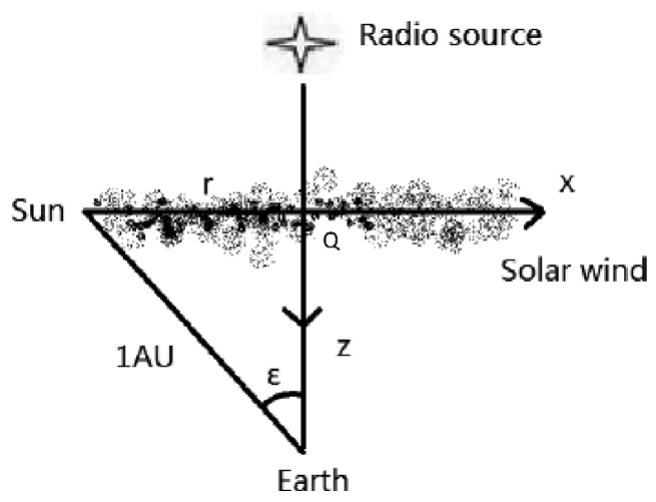

Figure 1. Geometry of IPS Concept.

\section{Theory}

Fig. 1 shows the geometry of IPS. z-axis is along the line-of-sight, $x$-axis is in the direction perpendicular to the z-axis pointing away from the Sun, and the y-axis is normal to the paper. $\mathrm{Q}$ is the point closest to the Sun along the line-of-sight, and $\varepsilon$ is the elongation angle, Sun-Earth-source. $\mathrm{r}$ is the distance between the Sun and Q, $r=\sin (\varepsilon)$ AU.There are two modes of IPS observations: Single-Station Single-Frequency (SSSF) mode and Single Station Dual-Frequency (SSDF) mode. The SSSF mode is easier to carry out and has been widely used. The observing system and data processing system 
for SSDF mode is more complicated, but it can measure the solar wind speed more accurately.

\section{Observations}

Since the discovery of IPS in 1964, many countries began to observe this phenomenon. China began IPS studies from the 1990s with the phased array mode of the Miyun Synthesis Radio Telescope (MSRT) at $232 \mathrm{MHz}$. Located at Miyun observatory in Beijing, it used the SSSF mode. From 2008, a new SSSF mode IPS observation system was built in Urumqi with the $25 \mathrm{~m}$ radio telescope in Xinjiang. Recently a new IPS observation system using the $50 \mathrm{~m}$ parabolic radio telescope located in Miyun, which is based on the SSDF mode at $\mathrm{S} / \mathrm{X}$ and UHF bands, is under construction to serve the National Meridian Project of China. Fig. 2 shows the the three stages of IPS observations in China.

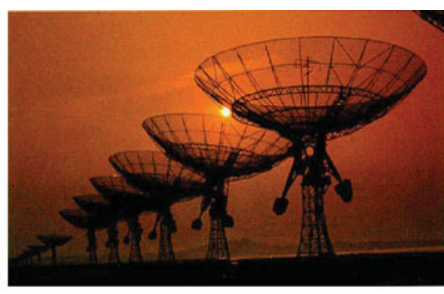

(a1)

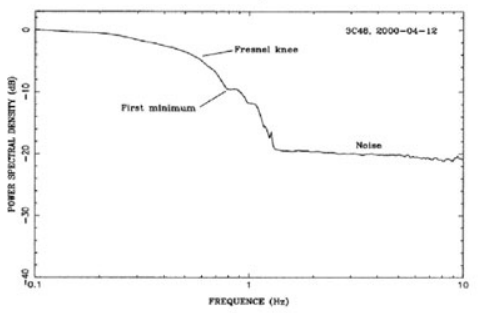

(a2)

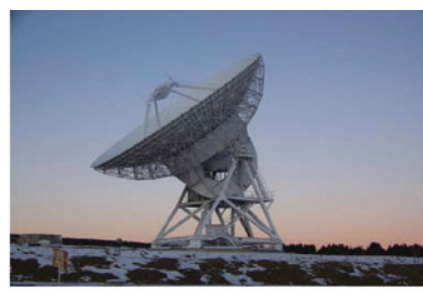

(b1)

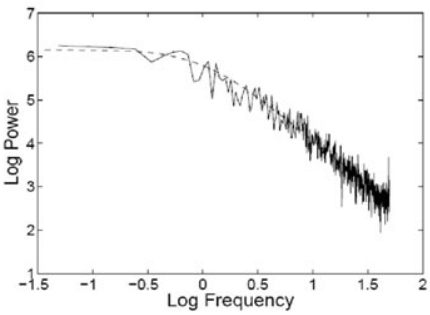

(b2)

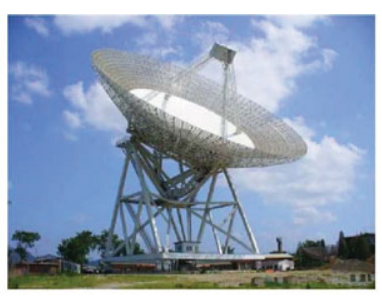

(c1)

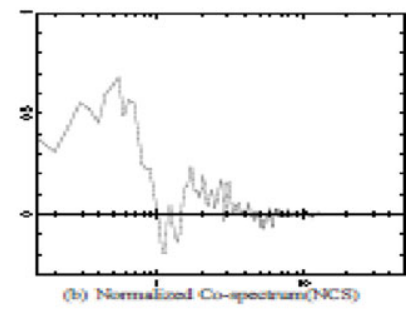

(c2)

Figure 2. Three stages of IPS observations in China. (a1) picture of MSRT, (a2) IPS power spectrum with MSRT; (b1) picture of $25 \mathrm{~m}$ telescope in Urumqi, (b2) IPS power spectrum with $25 \mathrm{~m}$; (c1) picture of $50 \mathrm{~m}$ telescope in Miyun, (c2) IPS power spectrum with $50 \mathrm{~m}$

\section{Conclusion}

IPS observations have been carried out in China for decades, and some achievements have been made(Wu et al. 2001; Liu et al. 2010; Zhang 2007). Nowadays human activity in the outer space is becoming more and more active. So the forecasting of space weather is much more important than in the past. IPS observations gives us an effective way to monitoring the Sun and solar wind. The IPS observation in China is still under developing, and the observation will trying to be a routine observation in the future.

\section{References}

Armstrong, J. W. \& Coles, W. A. 1972, J. Geophys. Res., 77, 4602

Hewish, A. \& Symonds, M. D. 1969, Planetary and Space Science, 17, 313

Liu, L. J., Zhang, X. Z., Li, J. B., Manoharan, P. K., Liu, Z. Y., \& Peng,B. 2010, RAA, 6, 577

Wu, J. H., Zhang, X. Z., \& Zheng, Y. J. 2001, Ap $6 S S$, 278, 189

Zhang, X. Z. 2007, CJAA, 5, 712 